Pacific Journal of Mathematics

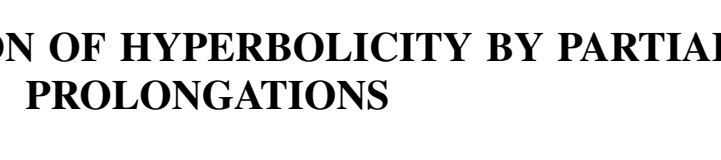




\title{
DETERMINATION OF HYPERBOLICITY BY PARTIAL PROLONGATIONS
}

\author{
H. H. JOHNSON
}

In [3] it was shown that non-hyperbolic systems of partial differential equations may sometimes be altered by partial prolongations so they become hyperbolic. This paper solves two problems concerning this process for normal systems with two independent variables. First, if hyperbolicity is obtainable, it can be obtained after a bounded number of steps, the bound depending only on the algebraic structure of the given system and easily calculated. Second, an explicit procedure is described whereby any system which is absolutely equivalent to a hyperbolic system can be changed into a hyperbolic system. In addition much of the underlying algebraic structure of such systems and their partial prolongations is analyzed.

These problems are local in nature, so we generally use local coordinate expressions. Readers familiar with fibre bundle and jet terminology will easily see how to express many concepts in the language of modern differential geometry. All functions are assumed infinitely differentiable.

1. Systems. Let $\sum$ denote a system of quasi-linear partial differential equations in dependent variables $z^{1}, \cdots, z^{m}$ and two independent variables $x^{1}, x^{2}$ which can be solved for the partials $\partial z^{\lambda} / \partial x^{2}=\partial_{2} z^{\lambda}$ :

$$
\Sigma\left\{\begin{array}{l}
\partial_{2} z^{\lambda}=A_{\mu}^{\lambda} \partial_{1} z^{\prime \prime}+B^{\lambda}, \lambda=1, \cdots, m, \\
\Theta^{\alpha}=C_{\lambda}^{\alpha} \partial_{1} z^{\lambda}+D^{\alpha}=0, \alpha=1, \cdots, \alpha_{1}, \\
f^{\beta}\left(x^{2}, z^{\lambda}\right)=0, \beta=1, \cdots, \beta_{1} .
\end{array}\right.
$$

Here we use the summation convention, $\partial_{1} z^{\lambda}=\partial z^{\lambda} / \partial z^{1}$, and $A_{i}^{\lambda}, B^{\lambda}, C_{\lambda}^{\alpha}$, $D^{\alpha}, f^{\beta}$ are (infinitely differentiable) functions of $x^{1}, x^{2}, z^{1}, \cdots, z^{m}$, on some open set $U$ in $R^{m+2}$.

The following notation will be useful. If $F=F\left(x^{1}, x^{2}, z^{1}, \cdots, z^{m}\right.$, $\left.\partial_{1} z^{1}, \cdots, \partial_{1} z^{m}\right)$ is a function on an open set $V$ of $R^{2 m+2}$, then for $\left(x^{1}\right.$, $\left.x^{2}, z^{1}, \cdots, z^{m}\right)$ in $\mathrm{U}$, we define

$$
\begin{aligned}
\partial_{1}^{*} F= & F_{x^{1}}+F_{z^{\prime}} \partial_{1} z^{\lambda}+F_{\partial_{1} z^{z}} \partial_{11} z^{\lambda}, \\
\partial_{2}^{*} F= & F_{x^{2}}+F_{z^{\lambda}}\left[A_{\mu}^{\lambda} \partial_{1} z^{\mu}+B^{\lambda}\right] \\
& +F_{\partial_{1} z^{z}} \partial_{1}^{*}\left[A_{\mu}^{\lambda} \partial_{1} z^{\prime \prime}+B^{\lambda}\right] .
\end{aligned}
$$

These are functions of $x^{1}, x^{2}, z^{1}, \cdots, z^{m}, \partial_{1} z^{1}, \cdots, \partial_{1} z^{m}, \partial_{11} z^{1}, \cdots, \partial_{11} z^{m}$ where $\partial_{11} z^{\lambda}=\partial^{2} z^{2} / \partial x^{1} \partial x^{1}$. 
DEFINITION 1. In this paper the collection $\Sigma$ of equations will be called a system when there are equations

$$
\begin{gathered}
\partial_{2}^{*} \Theta^{\alpha}=R_{\gamma}^{\alpha} \partial_{1}^{*} \Theta^{\gamma}+S_{\gamma}^{\alpha} \Theta^{\gamma}, \\
\partial_{2}^{*} f^{\beta}=T_{\alpha}^{\beta} \Theta^{\alpha}, \quad \partial_{1}^{*} f^{\beta}=U_{\alpha}^{\beta} \Theta^{\alpha},
\end{gathered}
$$

$\alpha=1, \cdots, \alpha_{1} ; \beta=1, \cdots, \beta_{1}$; where $R_{\gamma}^{\alpha}, S_{\gamma}^{\alpha}, T_{\alpha}^{\beta}, U_{\alpha}^{\beta}$ are functions of $x^{i}, z^{\lambda}$ on $U$. Then $U$ is the domain of $\Sigma$. If $z^{\lambda}=\varphi^{\lambda}\left(x^{1}\right), x_{0}^{1} \leqq x^{1} \leqq x_{1}^{1}$ is any solution of $\Theta^{\alpha}=0$ for fixed $x_{0}^{2}$, where $f^{\beta}\left(x_{0}^{1}, x_{0}^{2}, \varphi^{\lambda}\left(x_{0}^{1}\right)\right)=0$, this 1-dimensional solution may be extended to a full solution by solvingthe initial value problem $\partial_{2} z^{\lambda}=A_{\mu}^{\lambda} \partial_{1} z^{\mu}+B^{\lambda}, \lambda=1, \cdots, m, z^{\lambda}\left(x^{1}, x_{0}^{2}\right)=$ $\varphi^{\lambda}\left(x^{\prime}\right)$. Such systems are called "involutive".

We now define for each point $(x, z)$ in $U$ an associated vector space $V(x, z)$ to be that vector space over $R$ with basis $\partial_{1} z^{1}, \cdots, \partial_{1} z^{m}$. We also define an associated linear transformation $A(x, z)$ :

$$
V(x, z) \rightarrow V(x, z) \text { defined by } A(x, z)\left(\partial_{1} z^{\lambda}\right)=A_{\mu^{\prime}}^{2}(x, z) \partial_{1} z^{\mu} .
$$

A vector field on an open subset $W$ of $\mathbf{U}$ is a smooth assignment of a vector in the vector space at each point of $W$, that is, $C_{\lambda}(x, z) \partial_{1} z^{\lambda}$ in $V(x, z)$ where $C_{\lambda}$ are infinitely differentiable on $W$.

Proposition 1. If $\Sigma$ is a system then the vector fields $C_{i}^{\alpha}(x, z) \partial_{1} z^{\lambda}, \alpha=1, \cdots, \alpha_{1}$, span a subspace of $V(x, z)$ which is invariant under $A(x, z)$.

Proof. Comparing coefficients of $\partial_{11} z^{\mu}$ in equations (1) leads to

$$
C_{\lambda}^{\alpha} A_{\mu}^{\lambda}=R_{\gamma}^{\alpha} C_{\mu^{\prime}}^{\gamma} \text { all } \alpha, \mu .
$$

Hence

$$
\begin{aligned}
A\left(C_{\lambda}^{\alpha} \partial_{1} z^{\lambda}\right) & =C_{\lambda}^{\alpha} A\left(\partial_{1} z^{\lambda}\right) \\
& =C_{\lambda}^{\alpha} A_{\hat{\lambda}}^{\lambda} \partial_{1} z^{\mu}=R_{\gamma}^{\alpha} C_{\mu}^{\lambda} \partial_{1} z^{\mu},
\end{aligned}
$$

at each point of $U$.

This subspace is the invariant subspace of $\Sigma$ at $(x, z)$ and denoted $C(x, z)$.

A change in the dependent variables of $\Sigma$ results in a similarity transformation on $A(x, z)$, corresponding to a basis change of $V(x, z)$. The invariant subspace $C(x, z)$ is preserved. Thus, $A, V$ and $C$ are invariant under a change in dependent variables.

Definition 2. A system $\Sigma_{1}$ in variables $x^{1}, x^{2}, z^{1}, \cdots, z^{m} u^{1}, \cdots, u^{p}$ is called a partial prolongation (which we abbreviate $p p$ ) of $\Sigma$ if the projection map $\pi(x, z, u)=(x, z)$ carries its domain $\bigcup_{1}$ onto the domain 
$\mathrm{U}$ of $\Sigma$ and if $\Sigma_{1}$ contains the equations of $\Sigma$ together with equations for each $u^{\pi}$, namely,

$$
u^{\pi}=E_{\lambda}^{\pi} \partial_{1} z^{\lambda}+F^{\pi}, \pi=1, \cdots, p,
$$

where $E_{\lambda}^{\pi}$ and $F^{\pi}$ depend only on $(x, z)$. Thus $\Sigma_{1}$ contains in addition to the equations of $\Sigma$ equations (3) and

$$
\partial_{2} u^{\pi}=F_{\rho}^{\pi} \partial_{1} u^{\rho}+G_{\lambda}^{\pi} \partial_{1} z^{\lambda}+H^{\pi}, \pi=1, \cdots, p .
$$

Finally, in order to satisfy the Definition 1 of a system (see equations (2)), if $L(x, z, u)=0$ is any equation which can be deduced from the equations of $\Sigma_{1}$, then $\partial_{1}^{*} L=0$ and $\partial_{2}^{*} L=0$ must be in $\Sigma_{1}$. The $p p$ is normal if it can be defined so the vector fields $E_{\lambda}^{\pi} \partial_{1} z^{\lambda}$ and $C_{\lambda}^{\alpha} \partial_{1} z^{\lambda}$ are linearly independent at each point of $U$. The invariant space associated with $\Sigma_{1}$ at $(x, z, u)$ is spanned by $C_{\lambda}^{\alpha}(x, z) \partial_{1} z^{\lambda}, \alpha=1, \cdots, \alpha_{1}$, and $E_{\lambda}^{\pi}(x, z) \partial_{1} z^{\lambda}, \pi=1, \cdots, p$.

Partial prolongations are the basic tool we shall use in modifying given systems. It was shown in [3] that there is a natural correspondence between the solutions of $\Sigma$ and $\Sigma_{1}$. This correspondence is what was behind Élie Cartan's use of the term 'absolute equivalence' for systems which could be related by a sequence of partial prolongations [1]. See [2] for a discussion of these general questions. We first explore some consequences of Definition 2.

Proposition 2. If at each point of $\bigcup$ the vectors $C_{\lambda}^{\alpha} \partial_{1} z^{\lambda}$, and $E_{\hat{\lambda}}^{\pi} \partial_{1} z^{\lambda}$ are linearly independent then $F_{\rho}^{\pi}$ and $S_{\alpha}^{\pi}$ depend only on $(x, z)$, not $u^{1}, \cdots, u^{p}$.

Proof. Applying condition (1) of Definition 1 to the equations $\Psi^{\pi}=u^{\pi}-E_{\lambda}^{\pi} \partial_{1} z^{\lambda}-F^{\pi}=0$ in $\Sigma_{1}$ gives $\partial_{2}^{*} \Psi^{\pi} \equiv 0$ modulo $\left(\partial_{1}^{*} \Psi^{\rho}, \partial_{1}^{*} \Theta^{\alpha}, \Psi^{\rho}\right.$, $\left.\Theta^{\alpha}\right)$, i.e.,

$$
\begin{aligned}
\partial_{2}^{*}\left[u^{\pi}-E_{\lambda}^{\pi} \partial_{1} z^{\lambda}-F^{\pi}\right]= & R_{\rho}^{\pi} \partial_{1}^{*}\left[u^{\rho}-E_{\lambda}^{\rho} \partial_{1} z^{\lambda}-F^{\rho}\right] \\
& +S_{\alpha}^{\pi} \partial_{1}^{*}\left[C_{\lambda}^{\alpha} \partial_{1} z^{\lambda}+D^{\alpha}\right] \\
& +T_{\rho}^{\pi}\left[u^{\rho}-E_{\lambda}^{\rho} \partial_{1} z^{\lambda}-F^{\rho}\right] \\
& +\bigcup_{\alpha}^{\pi}\left[C_{\lambda}^{\alpha} \partial_{1} z^{\lambda}+D^{\alpha}\right],
\end{aligned}
$$

for some $R_{\rho}^{\pi}, S_{\alpha}^{\pi}, T_{\rho}^{\pi}$ and $\bigcup_{\alpha}^{\pi}$. Comparing coefficients of $\partial_{1} u^{\rho}$ and $\partial_{11} z^{\lambda}$ we have $F_{\rho}^{\pi}=R_{\rho}^{\tau}$, and

$$
\begin{aligned}
-E_{\mu}^{\pi} A_{\lambda}^{\mu} & =-R_{\rho}^{\pi} E_{\lambda}^{\rho}+S_{\alpha}^{\pi} C_{\lambda}^{\alpha} \\
& =-F_{\rho}^{\pi} E_{\lambda}^{\rho}+S_{\alpha}^{\pi} C_{\lambda}^{\alpha} .
\end{aligned}
$$

Using the fact that $A_{\lambda}^{\mu}, E_{\lambda}^{\rho}$ and $C_{\lambda}^{\alpha}$ are functions of $(x, z)$ alone, if we take the partial derivative of (6) with respect to $u^{\sigma}$ we obtain $0=$ 
$-F_{\rho}^{\pi},{ }_{u^{o}} E_{\lambda}^{\rho}+S_{\alpha}^{\pi},{ }_{u^{o}} C_{\lambda}^{\alpha}$. The linear independence hypothesis now implies the result.

Proposition 3. If $\Sigma_{1}$ is a normal pp the invariant subspace $C_{1}(x, z, u)$ of $\Sigma_{1}$ at $(x, z, u)$ is independent of $u^{1}, \cdots, u^{p}$ and as $a$ subspace of $V(x, z)$ is invariant under $A(x, z)$.

Proof. Independence of $u^{1}, \cdots, u^{p}$ follows from Proposition 2. Invariance follows from equations (6).

Thus, the possible $p p$ 's of $\Sigma$ depend on the subspaces of $V(x, z)$ which are invariant under $A(x, z)$.

Definition 3. A change in dependent variables will mean the obvious system in new variables

$$
\bar{z}^{\lambda}=h^{\lambda}\left(x^{1}, x^{2}, z^{1}, \cdots, z^{m}\right), \lambda=1, \cdots, m
$$

defined on $U$ which must have inverse functions $z^{\lambda}=g^{\lambda}\left(x^{1}, x^{2}, \bar{z}^{1}, \cdots\right.$, $\left.\bar{z}^{m}\right)$. For example, in $\Sigma_{1}$ of Definition 2 we may replace $u^{\pi}$ by $\bar{u}^{\pi}=$ $u^{\pi}-F^{\pi}$. Then $\Sigma_{1}$ is a $p p$ on $\bar{u}^{\pi}=E_{\lambda}^{\pi} \partial_{1} z^{\lambda}$.

DEFINITION 4. If $\Sigma$ contains equations of the form

$$
z^{r+k}=f^{k}\left(x^{1}, x^{2}, z^{1}, \cdots, z^{r}\right), k=1, \cdots, m-r,
$$

then by Definition 1 it contains

$$
\begin{aligned}
& \partial_{2} z^{r+k}=\sum_{\lambda=1}^{r} f^{k},{ }_{z^{\lambda}}\left[A_{i}^{\lambda} \partial_{1} z^{\mu}+B^{\lambda}\right]+f^{k},{ }_{x^{2}}, \\
& \partial_{1} z^{r+k}=\sum_{\lambda=1}^{r} f^{k},{ }_{z^{\lambda}} \partial_{1} z^{\lambda}+f^{k},{ }_{x^{1}} \cdot
\end{aligned}
$$

The system $\bar{\Sigma}$ obtained from $\Sigma$ by replacing $z^{r+k}, \partial_{1} z^{r+k}, \partial_{2} z^{r+k}$ by their expressions in terms of $x^{1}, x^{2}, z^{1}, \cdots, z^{r}, \partial_{1} z^{1}, \cdots, \partial_{1} z^{r}$ is called an $a d-$ missible restriction of $\Sigma$.

Proposition 4. If $\Sigma_{1}$ is a pp of $\Sigma$ and $\Sigma_{0}$ is an admissible restriction of $\Sigma$, then there is an admissible restriction of $\Sigma_{1}, \Sigma_{10}$, which is a pp of $\Sigma_{0}$.

This follows from the definitions.

Proposition 5. If $\Sigma_{1}$ is a normal $p p$ of $\Sigma$ then the equations of $\Sigma_{1}$ are uniquely determined by the vector fields $E_{\lambda}^{\pi} \partial_{1} z^{\lambda}$ up to a change of dependent variables. That is, $G_{\lambda}^{\pi}$ and $H^{\pi}$ are uniquely determined up to arbitrary linear combinations of the equations $C_{\lambda}^{\alpha} \partial_{1} z^{\lambda}+D^{\alpha}=0$ 
and $E_{\lambda}^{\pi} \partial_{1} z^{\lambda}+F^{\pi}-u^{\pi}=0 . \quad\left(\right.$ We say then that $\Sigma_{1}$ is the pp on $E_{\lambda}^{\pi} \partial_{1} z^{\lambda}$.)

Proof. By changing variables make $F^{\pi}=0$. Since $E_{\lambda}^{\pi} \partial_{1} z^{\lambda}$ and $C_{\lambda}^{\alpha} \partial_{1} z^{\lambda}$ are linearly independent, $F_{\rho}^{\pi}$ and $S_{\alpha}^{\pi}$ are uniquely determined by equation (6). Comparing coefficients of $\partial_{1} z^{\lambda}$ in (5) using (4), we find

$$
\begin{aligned}
& G_{\lambda}^{\pi}-E_{\lambda}^{\pi},{ }_{x^{2}}-E_{\mu}^{\pi}\left(A_{\lambda}^{\mu},{ }_{x^{2}}+B^{\mu},{ }_{z^{\lambda}}\right) \\
& =-R_{\rho}^{\pi} E_{\lambda}^{\rho},{ }_{x^{1}}+S_{\alpha}^{\pi}\left(C_{\lambda}^{\alpha},{ }_{x^{1}}+D^{\alpha},{ }_{z^{\lambda}}\right)-T_{\rho}^{\pi} E_{\lambda}^{\rho} \\
& \quad+\bigcup_{\alpha}^{\pi} C_{\lambda}^{\alpha} .
\end{aligned}
$$

Thus, $G_{\lambda}^{\pi}$ is uniquely determined up to a linear combination of $E_{\lambda}^{\rho}$ and $C_{\lambda}^{\alpha}$. Comparing the terms (5) which are independent of $\partial_{1} z^{\lambda}$ leads to the same conclusion about $H^{\pi}$.

Proposition 6. Let $\Sigma_{1}$ be a normal pp of $\Sigma$. Let $V_{1}(x, z, u)$ and $V(x, z)$ be the vector spaces associated with $\Sigma_{1}$ and $\Sigma$, respectively. Let $C_{1}(x, z, u)=C_{1}(x, z)$ and $C(x, z)$ be the invariant subspaces of $\Sigma_{1}$ and $\Sigma$, respectively, and $A_{1}(x, z, u), A(x, z)$ their linear transformations. Then there exist natural horizontal exact sequences such that the following diagram is commutative:

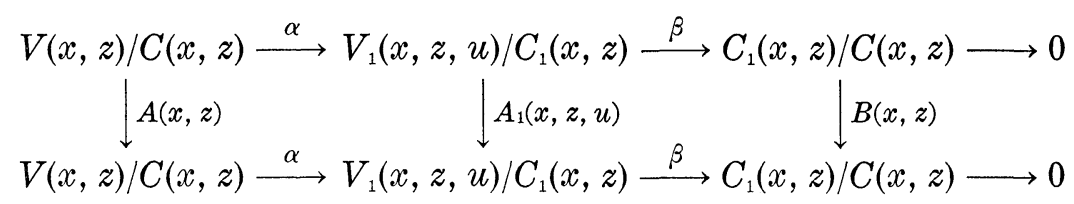

where

$$
\begin{aligned}
& \alpha\left(\partial_{1} z^{\lambda}+C(x, z)\right)=\partial_{1} z^{\lambda}+C_{1}(x, z), \\
& \beta\left(\partial_{1} z^{\lambda}+C_{1}(x, z)\right)=0+C(x, z), \\
& \beta\left(\partial_{1} u^{\pi}+c_{1}(x, z)\right)=E_{\lambda}^{\pi} \partial_{1} z^{\lambda}+C(x, z),
\end{aligned}
$$

and $B(x, z)$ is the natural linear transformation induced by $A(x, z)$.

Proof. Let us omit the dependence on $(x, z)$ or $(x, z, u)$. We have $A\left(\partial_{1} z^{\lambda}\right)=A_{\mu}^{\lambda} \partial_{1} z^{\mu}=A_{1}\left(\partial_{1} z^{\lambda}\right), A_{1}\left(\partial_{1} u^{\tau}\right)=F_{\rho}^{\pi} \partial_{1} u^{\rho}+G_{\lambda}^{\pi} \partial_{1} z^{\lambda}$. Then $C_{1} / C$ is spanned by $E_{\lambda}^{\pi} \partial_{1} z^{\lambda}+C$, where $A\left(E_{\lambda}^{\pi} \partial_{1} z^{\lambda}\right)=F_{\rho}^{\pi} E_{\lambda}^{o} \partial_{1} z^{\lambda}+C$. By definition $\beta \alpha=0$ and the kernel of $\beta$ is $V / C_{1}$ since the $E_{\lambda}^{\pi} \partial_{1} z^{\lambda}$ are independent modulo $C$. This kernel of $\beta$ is the image of $\alpha$. Hence exactness. Commutativity is routinely checked.

Definition 5. The eigenvalues of $\Sigma$ at $(x, z)$ are the eigenvalues of $A(x, z)$ on $V(x, z) / C(x, z)$.

Proposition 7. If $\Sigma_{1}$ is a normal pp of $\Sigma$ then the eigenvalues 
of $\Sigma_{1}$ at $(x, z, u)$ are those of $\Sigma$ at $(x, z)$.

Proof. Let $w^{\gamma}=W_{\lambda}^{\gamma} \partial_{1} z^{\lambda}$ fill out a basis of $V(x, z)$ together with $v^{\alpha}=C_{\lambda}^{\alpha} \partial_{1} z^{\lambda}$ and $u^{\pi}=E_{\lambda}^{\pi} \partial_{1} z^{\lambda}$. Suppose

$$
A\left(u^{\pi}\right)=F_{\rho}^{\pi} u^{\rho}+\widetilde{G}_{\alpha}^{\pi} v^{\alpha}, A\left(w^{\gamma}\right)=M_{\varepsilon}^{\gamma} w^{\varepsilon}+N_{\pi}^{\gamma} u^{\pi}+T_{\alpha}^{\gamma} v^{\alpha} .
$$

The eigenvalues of $\Sigma$ at $(x, z)$ are those of

$$
\left(\begin{array}{ll}
F & N \\
O & M
\end{array}\right)
$$

where $F=\left(F_{\rho}^{\pi}\right), N=\left(N_{\pi}^{r}\right), M=\left(M_{\varepsilon}^{r}\right)$.

If $y^{\pi}=\partial_{1} u^{\pi}$, then $A_{1}\left(y^{\pi}\right)=F_{\rho}^{\pi} y^{\rho}+P_{\gamma}^{\pi} w^{\gamma}+Q_{\rho}^{\pi} u^{\rho}+L_{\alpha}^{\pi} v^{\alpha}$, so the eigenvalues of $\Sigma_{1}$ on $V_{1} / C_{1}$ are those of the

$$
\left(\begin{array}{cc}
M & P \\
O & F
\end{array}\right)
$$

where $P=\left(P_{r}^{\pi}\right)$.

We have found that a normal $p p$ of $\Sigma$ is completely determined by vector fields $E_{\lambda}^{\pi} \partial_{1} z^{\lambda}$ which together with $C_{\lambda}^{\alpha} \partial_{1} z^{\lambda}$ span a subspace of $V(x, z)$ which is invariant under $A(x, z)$. However, there need not be $p p$ 's on any given invariant vector fields. This is because of the equations which must be satisfied in (5) for the coefficients of $\partial_{1} z^{\lambda} \partial_{1} z^{\mu}$ :

$$
\begin{aligned}
-E_{\lambda}^{\pi},{ }_{z^{\nu}} A_{\mu^{\mu}}^{\nu}-E_{\mu \nu}^{\pi},{ }_{{ }^{\nu}} A_{\lambda}^{\nu}= & -R_{\rho}^{\pi}\left(E_{\lambda}^{\rho},{ }_{z^{\mu}}+E_{\mu}^{\rho},{ }_{z^{\lambda}}\right) \\
& -S_{\alpha}^{\pi}\left(C_{\lambda}^{\alpha},{ }_{z^{\mu}}+C_{\mu}^{\alpha},{ }_{z^{\lambda}}\right) .
\end{aligned}
$$

One situation when these equations are always satisfied is when the entire space $V(x, z)$ is used for the $p p$.

Definition 6. The total prolongation (abbreviated tp) $P \Sigma$ of $\Sigma$ is obtained as the $p p$ on vector fields which span all of $V(x, z)$ at each point.

Proposition 8. The tp of $\Sigma$ always exists.

Proof. The system $P \Sigma$ is variables $x^{i}, z^{2}, p^{1}, \cdots, p^{m}$ is obtained by adding to the equations of $\Sigma$ the equations

$$
\begin{aligned}
p^{\lambda}= & \partial_{1} z^{\lambda}, \\
\partial_{2} p^{\lambda}= & A_{\mu}^{\lambda} \partial_{1} p^{\mu}+\left[A_{\mu}^{\lambda},{ }_{z^{\nu}}\left(A_{\omega}^{\nu} p^{\omega}+B^{\nu}\right)+A_{\mu}^{\lambda},{ }_{x^{2}}\right] p^{\mu} \\
& +B^{\lambda},{ }_{z^{\mu}} p^{\mu}+B^{\lambda},{ }_{x^{2}}, \lambda=1, \cdots, m, \\
C_{\lambda}^{\alpha} \partial_{1} p^{\lambda} & +\left(\partial_{1}^{*} C_{\lambda}^{\alpha}\right) p^{\lambda}+\partial_{1}^{*} D^{\alpha}=0, \alpha=1, \cdots, \alpha_{1} .
\end{aligned}
$$


The last set of equations is added because $p \Sigma$ contains $C_{\lambda}^{\alpha} p^{\lambda}+D^{\alpha}=0$ in order to satisfy conditions (2) in Definition 1 for functional equations in systems. It is a calculation to check that equations (7) together with those in $\Sigma$ constitute a system.

The $t p$ is not normal, but using the equations $C_{\lambda}^{\alpha} p^{\lambda}+D^{\alpha}=0$ it is possible to find an admissible restriction of $P \Sigma$ which is a normal $p p$ of $\Sigma$. The invariant subspace associated with $P \Sigma$ is spanned by $\partial_{1} z^{1}, \cdots, \partial_{1} z^{m}$ and $C_{\lambda}^{\alpha} \partial_{1} p^{\lambda}, \alpha=1, \cdots, \alpha_{1}$.

Proposition 9. Let $V, C$ and $A$ denote the vector space, invariant subspace and linear transformation associated with $\Sigma$ at a point $(x, z)$. Let $P V, P C$ and $P A$ denote the same entities for $P \Sigma$ at $(x, z, p)$. There is a natural isomorphism $\pi: V / C \rightarrow P V / P C$ satisfying $\pi A=$ $(P A) \pi$. In particular subspaces of $P V / P C$ invariant under $P A$ correspond in a one-to-one way to those of $V / C$ invariant under $A$.

Proof. Let $\pi\left(\partial_{1} z^{\lambda}+C\right)=\partial_{1} p^{\lambda}+P C$. The rest is a calculation.

Proposition 10. If $\Sigma_{0}$ is an admissible restriction of $\Sigma$, then $P \Sigma_{0}$ is an admissible restriction of $P \Sigma$.

This follows from a calculation. The next result shows an important use of $t p$ 's.

Proposition 11. Let $E_{k}^{\pi} \partial_{1} z^{\lambda}, \pi=1, \cdots, p$, be vector fields on the domain of $\Sigma$ which, together with $C_{\lambda}^{\alpha} \partial_{1} z^{\lambda}$, span a subspace of $V(x, z)$ at each point which is invariant under $A(x, z)$. Then $E_{\lambda}^{\pi} \partial_{1} p^{\lambda}=$ $\pi\left(E_{\hat{\lambda}}^{\pi} \partial_{1} z^{\lambda}\right)$ satisfy a similar condition in $P V(x, z, p)$. Moreover, there always exists a pp of $P \Sigma$ on the vector fields $E_{\lambda}^{\pi} \partial_{1} p^{\lambda}$.

$$
\begin{aligned}
& \text { Proof. If } A\left(E_{\lambda}^{\pi} \partial_{1} z^{\lambda}\right)=F_{\rho}^{\pi} E_{\lambda}^{\rho} \partial_{1} z^{\lambda}+S_{\alpha}^{\pi} C_{\lambda}^{\alpha} \partial_{1} z^{\lambda} \text {, let } \\
& u^{\pi}=E_{i}^{\pi} \partial_{1} p^{\bar{\lambda}}, \\
& \partial_{2} u^{\pi}=F_{\rho}^{*} \partial_{1} u^{\rho}+\left(\partial_{2}^{*} E_{i}^{\pi}\right) \partial_{1} \rho^{\lambda} \\
& +E_{\lambda}^{\pi}\left[\left(\partial_{1}^{*} A_{\mu^{\prime}}^{\lambda}\right) \partial_{1} p^{\mu}+p^{\mu} \partial_{1}^{*}\left(\partial_{2}^{*} A_{\mu}^{\lambda}\right)+\left(\partial_{2}^{*} A_{\mu}^{\lambda}\right) \partial_{1} p^{\mu}+\partial_{1}^{*}\left(\partial_{2}^{*} B^{\lambda}\right)\right] \\
& -F_{\rho}^{\bar{\rho}}\left(\partial_{1}^{*} E_{i}^{\rho}\right) \partial_{1} p^{\lambda} \text {. }
\end{aligned}
$$

Observe that $\partial_{1}^{*} A_{\mu}^{\lambda}, \partial_{i}^{*} A_{k}^{\lambda}, \partial_{2}^{*} B^{\lambda}, \partial_{1}^{*} E_{\lambda}^{\rho}$ can be expressed in terms of $x, z, p$ using $\partial_{1} z^{\lambda}=p^{\lambda}$, while $\partial_{1}^{*}\left(\partial_{2}^{*} A_{\mu}^{\lambda}\right)$ and $\partial_{1}^{*}\left(\partial_{2}^{*} B^{\lambda}\right)$ are linear in $\partial_{1} p^{\nu}$. Consequently the above equation is linear in $\partial_{1} p^{2}$.

Proposition 12. Let $\Sigma_{1}$ be a normal pp of $\Sigma$ on $E_{\lambda}^{\pi} \partial_{1} z^{\lambda}, \pi=1, \cdots, p$. Let $(P \Sigma)_{1}$ be the pp of $P \Sigma$ on the $E_{\lambda}^{\pi} \partial_{1} p^{2}$. Then $(P \Sigma)_{1}$ is an admissible restriction of $P \Sigma_{1}$ and is a normal pp of $P \Sigma$. 
This is merely a calculation.

CoROLlaRY. If $\Sigma_{0} \subset \Sigma_{1} \subset \cdots \subset \Sigma_{h}$ is a sequence of systems where each $\Sigma_{i}$ is a normal pp of $\Sigma_{i-1}, i=1, \cdots, h$, then there exists a sequence $\Sigma_{0}^{\prime}=P \Sigma_{0} \subset \Sigma_{1}^{\prime} \subset \cdots \subset \Sigma_{h}^{\prime}$ where $\Sigma_{i}^{\prime}$ is a normal pp of $\Sigma_{i-1}^{\prime}$ and $P \Sigma_{i}$ has $\Sigma_{i}^{\prime}$ as an admissible restriction, $i=1, \cdots, h$.

Proposition 13. If $\Sigma_{1}$ and $\Sigma_{2}$ are pp's of $\Sigma$, then $\Sigma_{1} \cup \Sigma_{2}$ determines a pp of $\Sigma, \Sigma_{1}$ and $\Sigma_{2}$.

Proof. All that needs to be added to $\Sigma_{1} \cup \Sigma_{2}$ are equations $\partial_{1}^{*} L=$ $0, \partial_{2}^{*} L=0$ for any equation $L(x, z)=0$ which can be deduced from the equations in $\Sigma_{1}$ and $\Sigma_{2}$.

Proposition 14. If $\Sigma_{1}$ is a pp of $\Sigma$ using vector fields which lie in the invariant subspace $C(x, z)$ of $\Sigma$ at each point, then $\Sigma_{1}$ has $\Sigma$ as an admissible restriction.

Proof. Suppose $\Sigma_{1}$ contains

$$
u^{\pi}=E_{\lambda}^{\pi} \partial_{1} z^{\lambda}=K_{\alpha}^{\pi} C_{\lambda}^{\alpha} \partial_{1} z^{\lambda} .
$$

Then $\Sigma_{1}$ contains the equations

$$
u^{\pi}=-K_{\alpha}^{\pi} D^{\alpha}
$$

so the $u^{\pi}$ may be eliminated by an admissible restriction (see Definition 4).

\section{Hyperbolicity.}

DEFINITION 7. Suppose that in a neighborhood of each point in the domain of $\Sigma$ there exist linearly independent vector fields $v^{\pi}=v_{\lambda}^{\pi}(x, z) \partial_{1} z^{\lambda}$, $\pi=1, \cdots, m-\alpha_{1}$, which together with $C_{\lambda}^{\alpha} \partial_{1} z^{\lambda} \operatorname{span} V(x, z)$ at each point. Suppose further that

$$
A_{\mu^{\mu}}^{\lambda}(x, z) v_{\hat{\lambda}}^{\bar{\lambda}}(x, z) \equiv K^{\pi} v_{\mu}^{\pi}(x, z)
$$

modulo $C(x, z)$. That is, $v^{\pi}+C$ is an eigenvector of $A$ on $V / C$ at each point with eigenvalue $K^{-}$. Then $\Sigma$ is called hyperbolic.

Definition 8. Let there exist a sequence $\Sigma \subset \Sigma_{1} \subset \Sigma_{2} \subset \cdots \subset \Sigma_{h}$ of systems in which each system is a $p p$ of the preceding system and in which $\Sigma_{h}$ is hyperbolic. Then $\Sigma$ is absolutely equivalent to a hyperbolic system.

In this event $\Sigma$ has the same behavior as a hyperbolic system [3].

Proposition 15. Hyperbolicity is preserved under admissible restrictions and total prolongations. 
Proof. If $z^{r+\lambda}=f^{r+\lambda}\left(x^{1}, x^{2}, z^{1}, \cdots, z^{r}\right), \lambda=1, \cdots, m-r$, define an admissible restriction, then $C(x, z)$ contains $\partial_{1} z^{r+\lambda}-\partial_{1}^{*} f^{r+\lambda}=0$. Hence $v^{\pi}$ must be linearly independent when $z^{r+\lambda}$ and $\partial_{1} z^{r+\lambda}$ are replaced by $f^{r+\lambda}$ and $\partial_{1}^{*} f^{r+\lambda}$, respectively. The second assertion follows from Proposition 9.

Proposition. 16. If $\Sigma$ is absolutely equivalent to a hyperbolic system the eigenvalues of $\Sigma$ are real at each point.

Proof. Proposition 7.

DeFinition 9. A vector $v \in V(x, z)$ is essential if for some eigenvalue $\lambda$ of $\Sigma$ at $(x, z)$ there is a vector $u \in V(x, z)$ such that

(1) $[A(x, z)-\lambda I] u \equiv v$,

(2) $[A(x, z)-\lambda I]^{s} v \equiv 0, s \geqq 1$,

(3) $v \neq 0$ modulo $C(x, z)$.

(Here $I$ is the identity.) The essential vectors belonging to an eigenvalue $\lambda$ generate a vector space $V_{\lambda}(x, z) \supset C(x, z)$. Let

$$
\rho(\Sigma)(x, z)=\sum_{\lambda} \operatorname{dim} V_{\lambda}(x, z) / C(x, z)
$$

be called the degeneracy of $\Sigma$ at $(x, z)$.

When $\Sigma$ is hyperbolic, $\rho(\Sigma)=0$ at all points.

THEOREM 1. Let $\Sigma \subset \Sigma_{1} \subset \cdots \subset \Sigma_{h}$ be a sequence of normal pp's leading to a hyperbolic system $\Sigma_{h}$. Let

$$
(x, z),\left(x, z, u_{1}\right), \cdots,\left(x, z, u_{1}, \cdots, u_{h}\right)
$$

be points in their domains, let $V_{j}\left(x, z, u_{1}, \cdots, u_{j}\right) \supset C_{j}\left(x, z, u_{1}, \cdots, u_{j}\right)$ be the associated and invariant spaces of $\Sigma_{j}$, where

$$
V(x, z) \subset V_{1}\left(x, z, u_{1}\right) \subset \cdots \subset V_{h}\left(x, z, u_{1}, \cdots, u_{h}\right) .
$$

Then if $v \in V(x, z)$ is an essential vector of $\Sigma, v \in C_{h}\left(x, z, u_{1}, \cdots, u_{h}\right)$.

Proof. By Proposition 6 , if $A_{h}\left(x, z, u_{1}, \cdots, u_{h}\right)$ is the linear transformation of $\Sigma_{h}$, then $A_{h}\left(x, z, u_{1}, \cdots, u_{h}\right) \mid V(x, z) \equiv A(x, z)$ modulo $C(x, z)$. Hence $v$ satisfies (1) and (2) of Definition 9 as an element in $V_{h}$. Being hyperbolic $\Sigma_{h}$ can have no essential vectors, so $v \in C_{h}$.

Proposition 17. Let $\Sigma \subset \Sigma_{1} \cdots \subset \Sigma_{h}$ be a sequence of normal pp's where $\Sigma_{h}$ is hyperbolic. Let $v^{\pi}=v_{\lambda}^{\pi} \partial_{1} z^{\lambda}$ be linearly independent vector fields of $\Sigma$ modulo $C(x, z)$ which are essential and which generate a subspace of $V(x, z) / C(x, z)$ invariant under $A(x, z)$. Let $\bar{\Sigma}$ be the pp of $\Sigma$ on the vector fields $v^{\pi}$. Then there exists a sequence of pp's $\Sigma \subset \bar{\Sigma} \subset \bar{\Sigma}_{1} \cdots \subset \bar{\Sigma}_{h}$ where $\bar{\Sigma}_{1}$ is a pp of $\Sigma$ and $\bar{\Sigma}_{h}$ has $\Sigma_{h}$ as an admissible restriction, hence is hyperbolic. 
Proof. Set $\bar{\Sigma}_{j}=\Sigma_{j} \cup \bar{\Sigma}$ and use Proposition 13 repeatedly. The hyperbolicity of $\bar{\Sigma}_{h}$ follows from Theorem 1 and Proposition 14 .

As a result of this proposition it can be supposed that any sequence of $p p$ 's leading to a hyperbolic system begins with a $p p$ on essential vectors. We shall show that this sort of $p p$ is the optimal way of obtaining hyperbolicity. We next examine more closely those essential vectors.

Definition 10. A system $\Sigma$ has a Jordan basis if there exist on its domain vector fields

$$
U_{k}^{s}=U_{k \lambda}^{s}(x, z) \partial_{1} z^{\lambda}, \quad 1 \leqq s \leqq m_{k}, \quad k=1, \cdots, r
$$

and eigenvalues $\lambda_{k}(x, z)$ of $A(x, z)$ modulo $C(x, z)$ such that

$$
\begin{aligned}
& {\left[A(x, z)-\lambda_{k}(x, z) I\right] u_{k}^{1}(x, z) \equiv 0 \text { modulo } C(x, z),} \\
& {\left[A(x, z)-\lambda_{k}(x, z) I\right] u_{k}^{s}(x, z) \equiv u_{k}^{s-1}(x, z) \text { modulo } C(x, z),}
\end{aligned}
$$

for $2 \leqq s \leqq m_{k}, 1 \leqq k \leqq r$, and these vector fields determine a basis of $V(x, z) / C(x, z)$. We also assume the functions $\lambda_{k}(x, z)$ are infinitely differentiable.

Proposition 18. Suppose $\Sigma$ has a Jordan basis $u_{k}^{s}$. Then a vector in $V(x, z)$ is essential with respect to an eigenvalue $\mu$ if and only if it is a linear combination modulo $C(x, z)$ of those $u_{k}^{s}(x, z)$ where $\lambda_{k}(x, z)=\mu, m_{k}>1$ and $1 \leqq s \leqq m_{k}-1$. Moreover, $\rho(\Sigma)(x, z)=$ $\sum_{k=1}^{r}\left(m_{k}-1\right)$.

Proof. "If" is immediate. Suppose

$$
u \equiv \sum_{k=1}^{r} \sum_{s=1}^{m_{k}} \boldsymbol{a}_{s}^{k} u_{k}^{s} \text { modulo } C
$$

is essential at $(x, z)$. Now, observe that

$$
\begin{aligned}
(A-\mu I)^{h} u_{k}^{s} \equiv & \left(\lambda_{k}-\mu\right)^{h} u_{k}^{s}+\left(\begin{array}{c}
h \\
1
\end{array}\right)\left(\lambda_{k}-\mu\right)^{h-1} u_{k}^{s-1}+\cdots \\
& +\left(\begin{array}{c}
h \\
j
\end{array}\right)\left(\lambda_{k}-\mu\right)^{h-j} u_{k}^{s-j}+\cdots+u_{k}^{s-h},
\end{aligned}
$$

so

$$
(A-\mu I)^{h} u \equiv \sum_{k=1}^{r} a_{m_{k}}^{k}\left(\lambda_{k}-\mu\right)^{h} u_{k}^{m_{k}}+L
$$

where $L$ involves only $u_{k}^{s}$ for $s \leqq m_{k}-1$. Hence, if $(A-\mu I)^{h} u \equiv 0$, $a_{m_{k}}^{k}=0$ whenever $\lambda_{k} \neq \mu$. Then

$$
(A-\mu I)^{h} u \equiv \sum_{k} a_{m_{k}-1}^{k}\left(\lambda_{k}-\mu\right)^{h} u_{k}^{m} k^{-1}+L^{\prime}
$$


where $L^{\prime}$ involves $u_{k}^{s}, s \leqq m_{k}-2$. Again these coefficients $a_{m_{k}-1}^{k}=0$ unless $\lambda_{k}=\mu$. Continuing we find $u$ depends only on $u_{k}^{s}, 1 \leqq s \leqq m_{k}$, $\lambda_{k}=\mu$. But $u \equiv(A-\mu I) v$, so this same result must hold for $v$. This implies that $u$ depends only on $u_{k}^{s}, 1 \leqq s \leqq m_{k}-1$.

Proposition 19. If $\Sigma$ has real eigenvalues, then there exists a dense open set $V$ in its domain such that every point of $V$ has a neighborhood on which $\Sigma$ has a Jordan basis and the invariant subspace $C$ is spanned by linearly independent vector fields.

Proof. This is a matter of examining various systems of linear and polynomial equations with coefficients which are functions of $(x, z)$.

DEFINITION 11. If each point in the domain of $\Sigma$ has a neighborhood on which the $C_{\lambda}^{\alpha} \partial_{1} z^{\lambda}$ are linearly independent and on which a Jordan basis can be found, let $E \Sigma$ be the $p p$ of $\Sigma$ on the essential vector fields. This is called the essential $p p$ of $\Sigma$. (Note that $E \Sigma$ may be defined locally. By Proposition 5 two $p p$ 's on local essential vector fields must coincide on the overlapping neighborhoods.)

We recall that $E \Sigma$ need not exist due to quadratic conditions, but by Proposition 9 the essential vector fields of $\Sigma$ and $P \Sigma$ correspond. By Proposition 11, there always exists a $p p$ on these vector fields which come from $\Sigma$. Thus we prove

Proposition 20. Any system $\Sigma$ may be restricted to a dense open set in its domain on which EP $\Sigma$ exists as a normal pp of $P \Sigma$. Similarly for $E P^{k} \Sigma, k \geqq 1$.

Proposition 21. Let $\Sigma \subset \Sigma_{1} \subset \cdots \subset \Sigma_{h}$ be a sequence of normal pp's where $\Sigma_{h}$ is hyperbolic. Then on an open dense subset of the domain of $\Sigma$ there is a sequence of pp's

$$
E P \Sigma \subset \Sigma_{1}^{\prime} \subset \cdots \subset \Sigma_{h}^{\prime}
$$

where $\Sigma_{h}^{\prime}$ is hyperbolic.

Proof. Use corollary to Proposition 12, Proposition 20, and Proposition 17.

Proposition 22. Let $\Sigma \subset \Sigma_{1} \subset \cdots \subset \Sigma_{h}$ be a sequence of $p p$ 's. Suppose the vector space $V(x, z)$ associated with $\Sigma$ is contained in the invariant space $C_{h}\left(x, z, u_{1}, \cdots, u_{h}\right)$ associated with $\Sigma_{h}$. Then there exists a sequence

$$
\Sigma \subset P \Sigma \subset \Sigma_{2}^{\prime} \subset \cdots \subset \Sigma_{h}^{\prime}
$$


where $\Sigma_{h}^{\prime}$ has $\Sigma_{h}$ as an admissible restriction.

Proof. Let $\Sigma_{j}^{\prime \prime}=\Sigma_{j} \cup P \Sigma=\Sigma_{j} \cup\left(\Sigma_{j-1} \cup P \Sigma\right)$. Clearly $\Sigma_{1}^{\prime \prime}=\Sigma_{1} \cup$ $P \Sigma$ has $P \Sigma$ as an admissible restriction. The result follows from Proposition 13 and 14.

Proposition 23. Let $\Sigma_{1}$ be a normal $p p$ of $P^{k} \Sigma, k \geqq 1$, on vector fields with coefficients which are functions of $(x, z)$. Then the essential vectors of $\Sigma_{1}$ can be spanned by vector fields having coefficients which are functions of the variables of $P \Sigma$.

Proof. This is a tautology for $k=1$. For $k>1$, assuming the result for $k-1$ to be true, by Proposition $12 \Sigma_{1}$ is an admissible restriction of a $p p P \Sigma^{\prime}$ where $\Sigma^{\prime}$ is the normal $p p$ of $P^{k-1} \Sigma$ on the corresponding vector fields in $P^{k-1} \Sigma$. By induction the essential vectors of $\Sigma^{\prime}$, hence of $P \Sigma^{\prime}$, hence $\Sigma_{1}$, are spanned by vector fields with coefficients functions of the variables of $P \Sigma$.

Definition 12. A $p p \Sigma_{1}$ of $\Sigma$ is complete if $\Sigma_{1}$ has no essential vectors which involve only the variables of $\Sigma$, i.e., of the form $a_{\lambda} \partial_{1} z^{\lambda}$.

For example, $P \Sigma$ is a complete $p p$ of $\Sigma$.

THeOREM 2. Let $\Sigma \subset \Sigma_{1} \subset \cdots \subset \Sigma_{h}$ be a sequence of normal pp's where $\Sigma_{h}$ is hyperbolic. For any $k \geqq m-\alpha_{1}-\rho(\Sigma)+1$, there exists a sequence of pp's on an open dense subset of the domain of $\Sigma$,

$$
P^{k} \Sigma \subset E^{\prime} P^{k} \Sigma \subset \Sigma_{1}^{\prime} \subset \cdots \subset \Sigma_{h}^{\prime}
$$

where $\Sigma_{h}^{\prime}$ is hyperbolic, $\Sigma_{1}^{\prime}$ is a pp of $P^{k} \Sigma, E^{\prime} P^{k} \Sigma$ is normal complete pp of $P^{k} \Sigma$ and $\rho(\Sigma)$ is the maximum value of the degeneracy of $\Sigma$. (Recall $m=$ no of dependent variables $z^{\lambda}, \alpha_{1}=\operatorname{dim} C(x, z)$. Thus, $m-$ $\alpha_{1}=\operatorname{dim} V(x, z) / C(x, z)$.)

Proof. Let $u_{2}^{\pi}(x, z) \partial_{1} z^{\lambda}$ be vector fields which span the essential vectors of $\Sigma$ on an open set in the domain of $\Sigma$. By Proposition 9, $u_{\lambda}^{\pi} \partial_{1} p_{k}^{\lambda}$ span the essential vectors of $P^{k} \Sigma$, where $p_{k}^{\lambda}$ are the variables in $P^{k} \Sigma$ which are analogous to the $p^{2}$ in Proposition 9 . Since $k \geqq 1$, $E P^{k} \Sigma$ exists and is a normal $p p$ of $P^{k} \Sigma$ for a suitable restriction by Proposition 20.

Now, by the corollary to Proposition 12, there is a sequence of normal $p p$ 's $P \Sigma \subset \widetilde{\Sigma}_{1} \subset \cdots \subset \widetilde{\Sigma}_{h}$ where $\widetilde{\Sigma}_{h}$ is hyperbolic. Repeating, we get a similar sequence $P^{k} \Sigma \subset \Sigma_{1}^{*} \subset \cdots \subset \Sigma_{h}^{*}$ where $\Sigma_{h}^{*}$ is hyperbolic. By Proposition 21 there is a sequence of $p p^{\prime}$ s $P^{k} \Sigma \subset E P^{k} \Sigma \subset{\widetilde{\Sigma_{1}^{\prime}}}_{1} \subset \cdots \subset \widetilde{\Sigma}_{h}^{\prime}$ where $\widetilde{\Sigma}_{h}^{\prime}$ is hyperbolic and $\widetilde{\Sigma}_{1}^{\prime}$ is a $p p$ of $P^{K} \Sigma$. 
If $E P^{k} \Sigma$ is a complete $p p$ of $P^{k} \Sigma$, the theorem is proved. Otherwise there exist essential vector fields of $E P^{k} \Sigma$ of the form $v_{\lambda}^{\sigma} \partial_{1} p_{k}^{\lambda}$. By Proposition 23 the coefficients $v_{k}^{\sigma}$ can be supposed to be functions of the variables $\left(x, z, p_{1}\right)$ of $P \Sigma$. By further restricting the open dense domain if necessary we can assume the $v_{\lambda}^{\sigma} \partial_{1} p_{k}^{2}$ and $u_{\hat{\Sigma}}^{\sigma} \partial_{1} p_{k}^{2}$ are linearly independent. They determine an invariant subspace of $P^{k} \Sigma$ modulo $C_{k}$ (the invariant space associated with $P^{k} \Sigma$ ), because the $v_{\lambda}^{\sigma} \partial_{1} p_{k}^{\lambda}$ are determined by the intersection of the space of essential vectors in $E P^{k} \Sigma$ with the image of $V_{k}$ (the total space associated with $P^{k} \Sigma$ ) in $E P^{k} \Sigma$, at each point. That is, they are merely those essential vectors in $E P^{k} \Sigma$ which depend only on $\partial_{1} p_{k}^{\lambda}$, the variables of $P^{k} \Sigma$.

By Proposition 11 we can form the normal $p p$ of $P^{k} \Sigma$ on the vector fields $v_{k}^{\sigma} \partial_{1} p_{k}^{\lambda}$ and $u_{\lambda}^{\pi} \partial_{1} p_{k}^{\lambda}$ provided that $k \geqq 2$. But if $k=1$, that means $m-\alpha_{1}-\rho(\Sigma)=0$, so every vector in $V(x, z)$ would be essential, which is impossible by the very Definition 11 of essential.

Letting $\mathscr{E} P^{k} \Sigma$ denote this $p p$, we see that $\mathscr{E} P^{k} \Sigma$ is a $p p$ of $E P^{k} \Sigma$ on some of that system's essential vector fields. Applying Proposition 17 there exists a sequence

$$
P^{k} \Sigma \subset E P^{k} \Sigma \subset \mathscr{E} P^{k} \Sigma \subset{\widetilde{\Sigma_{1}^{\prime \prime}}}_{1} \subset \cdots \subset{\widetilde{\Sigma_{h}^{\prime \prime}}}^{\prime \prime}
$$

where $\widetilde{\Sigma}_{1}^{\prime \prime}$ is a $p p$ of $P^{k} \Sigma$ and ${\widetilde{\Sigma_{h}^{\prime \prime}}}_{h}$ is hyperbolic.

If $\rho(\Sigma)=m-\alpha_{1}-1$, all vectors of $P^{k} \Sigma$ are essential except one, which must have been added in forming $\mathscr{E} P^{k} \Sigma$. Hence $\mathscr{E} P^{k} \Sigma$ is an admissible restriction of $P^{k+1} \Sigma$ and is a normal complete $p p$ of $P^{k} \Sigma$. Otherwise $m-\alpha_{1}-\rho(\Sigma)+1 \geqq 3$ and so the process above may be repeated for any further essential vectors in $\mathscr{E} P^{k} \Sigma$ of the form $w_{\hat{\lambda}} \partial_{1} p_{k}^{\lambda}$ in the variables of $P^{k} \Sigma$.

This process must end since there are at least $\rho(\Sigma)+h$ linearly independent vector fields added at the $h^{t h}$ stage of the form $a_{\lambda} \partial_{1} p_{k}^{\lambda}$, so we must arrive at $P^{k+1} \Sigma$ for $h \leqq m-\alpha_{1}-\rho(\Sigma) \leqq k-1$.

REMARK. Observe that $E^{\prime} P^{k} \Sigma$ is explicitely constructed.

Proposition 24. If $k \geqq m-\alpha_{1}-\rho(\Sigma)+1, j \geqq 0$, then $P^{j} E^{\prime} P^{k} \Sigma$ has $E^{\prime} P^{k+j} \Sigma$ as an admissible restriction, where $E^{\prime} P^{k} \Sigma$ and $E^{\prime} P^{k+j} \Sigma$ are constructed as in Theorem 2.

Proof. This follows by applying Proposition 12 at each stage in the process described in Theorem 2 and using Proposition 9 repeatedly.

Proposition 25. Let $\Sigma \subset \Sigma_{1} \subset \cdots \subset \Sigma_{h}$ be a sequence of $p p$ 's. Then there exists a sequence $\Sigma \subset \Sigma_{1}^{\prime} \subset \cdots \Sigma_{h}^{\prime}$ of normal pp's on an open dense set of the domain of $\Sigma$ where $\Sigma_{j}^{\prime}$ is an admissible restriction of $\Sigma_{j}$. 
Proof. By restricting the domain if necessary, $\Sigma_{1}$ is obtained by vector fields linearly independent of $\Sigma$ and possibly some in $C(x, z)$. Use Proposition 14 to obtain $\Sigma_{1}^{\prime}$ and make the same admissible restriction in all succeeding systems. Repeat this reasoning starting with $\Sigma_{1}$, etc.

THEOREM 3. Let $\Sigma \subset E^{\prime} \Sigma \subset \Sigma_{1} \subset \cdots \subset \Sigma_{h}$ be a sequence of normal pp's leading to the hyperbolic system $\Sigma_{h}$. Let $E^{\prime} \Sigma$ be a complete $p p$ of $\Sigma$ and suppose $\Sigma_{1}$ is a pp of $\Sigma$. Then on an open dense set, either $\rho\left(E^{\prime} \Sigma\right)<\rho(\Sigma)$ or else there exists a sequence of normal pp's

$$
P \Sigma \subset \Sigma_{2}^{\prime} \subset \cdots \subset \Sigma_{h}^{\prime}
$$

of one shorter length, leading to a hyperbolic system $\Sigma_{h}^{\prime}$.

Proof. Let $u_{k}^{s}=u_{k \lambda}^{s}(x, z) \partial_{1} z^{\lambda}$ be a Jordan basis on an open set $\mathbf{U}$,

$$
1 \leqq s \leqq m_{k}, k=1, \cdots, r:\left(A-\lambda_{k} I\right) u_{k}^{s} \equiv u_{k}^{s-1},\left(A-\lambda_{k} I\right) u_{k}^{\prime} \equiv 0
$$

modulo $C(x, z)$. Let $W(x, z)$ be the subspace of $V(x, z)$ used in the $p p E^{\prime} \Sigma$, i.e., $W(x, z)$ is the invariant subspace of $E^{\prime} \Sigma$ by Proposition 3 . Since $\left\{u_{k}^{s} \mid m_{k}>1,1 \leqq s \leqq m_{k}-1\right\}$ are all essential, they are contained in $W(x, z)$.

Let $W_{\mu}(x, z)$ be the subspace of $W(x, z)$ associated with the eigenvalue $\mu(x, z)$ of $A(x, z)$, modulo $C(x, z)$. Then $W_{\mu}(x, z) \supset\left\{u_{k}^{s} \mid \lambda_{k}=\right.$ $\left.\mu, m_{k}>1,1 \leqq s \leqq m_{k}-1\right\}$, where $\lambda_{k}$ is the eigenvalue associated with $u_{k}^{s}$.

If $W_{\mu}(x, z)$ contains vectors independent of these vectors, then on an open dense set we can find a factor field of the form

$$
\sum_{\lambda_{k}=\mu} C_{k}(x, z) u_{k}^{m_{k}} \in W_{\mu}(x, z) .
$$

Let $m_{j}=\sup \left\{m_{k} \mid \lambda_{k}=\mu, C_{k}(x, z) \neq 0\right]$. Denoting

$$
\begin{aligned}
w_{j}^{m_{j}} & =\sum C_{k} u_{k}^{m_{k}}, \\
(A-\mu I)^{r} w_{j}^{m_{j}} & \equiv \sum_{m_{k} \geqq m_{j}-r} C_{k} u_{v_{j}}^{m_{j}-r}=w_{j}^{m_{j}-r},
\end{aligned}
$$

$r=1, \cdots, m_{j}-1$, we can replace $u_{j}^{s}$ by $w_{j}^{s}$ and obtain a new Jordan basis. Repeating this process we find that in a neighborhood of any point in an open dense set one can find a Jordan basis for $\Sigma$ of the form $w_{k}^{s}, 1 \leqq s \leqq m_{k}, 1 \leqq k \leqq r$ where for $1 \leqq s \leqq m_{k}, 1 \leqq k \leqq r^{\prime}$ and $1 \leqq s \leqq m_{k}-1, r^{\prime}<k \leqq r^{\prime \prime}$, these elements span $W(x, z)$. Moreover, $m_{k}=1$ if $k>r^{\prime \prime}$.

There are two cases.

Case 1. For some $m_{k}>1, w_{k}^{m_{k}} \notin W(x, z)$. Then $E^{\prime} \Sigma$ is obtained 
locally by a $p p$ of the form $z_{k}^{s}=w_{k \lambda}^{s} \partial_{1} z^{\lambda} ; 1 \leqq s \leqq m_{k}$ for $1 \leqq k<r^{\prime}$; $1 \leqq s \leqq m_{k}-1$ for $r^{\prime}<k \leqq r^{\prime \prime}$ where some $m_{k}>1$ for $r^{\prime}<k \leqq r^{\prime \prime}$.

$$
\partial_{2} z_{k}^{s}=\lambda_{k} \partial_{1} z_{k}^{s}+\partial_{1} z_{k}^{s-1}+\sum_{h=r^{\prime}+1}^{r} R_{k h}^{s} w_{h}^{m_{h}}+T_{k}^{s},
$$

for $s>1$,

$$
\partial_{2} z_{k}^{1}=\lambda_{k} \partial_{1} z_{k}^{1}+\sum_{h=r^{\prime}+1}^{r} R_{k h}^{1} w_{h}^{m} h+T_{k}^{s} .
$$

We shall show this system has lower degeneracy than $\Sigma$. The space $V^{\prime}\left(x, z^{\lambda}, z_{k}^{s}\right)$ associated with $E^{\prime} \Sigma$ is spanned by $\partial_{1} z^{\lambda}, \partial_{1} z_{k}^{s}$. Its invariant subspace $C^{\prime}\left(x, z^{\lambda}, z_{k}^{s}\right)$ is spanned by $C_{\lambda}^{\alpha} \partial_{1} z^{\lambda}$ together with $w_{k}^{s}, 1 \leqq s \leqq m_{k}$ for $1 \leqq k \leqq r^{\prime} ; 1 \leqq s \leqq m_{k}-1$ for $r^{\prime}<k \leqq r^{\prime \prime}$. Thus $V^{\prime} / C^{\prime}$ is spanned by $\partial_{1} z_{k}^{s}, w_{h}^{m_{h}}, h>r^{\prime}$. Set $t_{k}^{s}=\partial_{1} z_{k}^{s}+\sum_{h>r^{\prime}} f_{k r}^{s} w_{h}^{m_{h}}, 1 \leqq s \leqq m_{k}$ for $1 \leqq$ $k \leqq r^{\prime}$ and $1 \leqq s \leqq m_{k}-1$ for $r^{\prime}<k \leqq r^{\prime \prime}$, where for $\lambda_{h} \neq \lambda_{k}$,

$$
f_{k h}^{1}=\frac{R_{k h}^{1}}{\lambda_{l}-\lambda_{h}}, \quad f_{k h}^{s}=\frac{f_{k h}^{s-1}-R_{k h}^{s}}{\lambda_{k}-\lambda_{h}}
$$

and for $\lambda_{k}=\lambda_{h}, f_{k h}^{s}=R_{k h}^{s+1}$. Then

$$
\begin{aligned}
& \left(A^{\prime}-\lambda_{k} I\right) t_{k}^{s} \equiv t_{k}^{s-1} \quad \text { if } \quad s>1, \\
& \left(A^{\prime}-\lambda_{k} I\right) t_{k}^{1} \equiv \sum_{\lambda_{h}=\lambda_{k, h}>r^{\prime}} R_{k h}^{1} w_{h}^{m_{h}}=t_{k}^{0} .
\end{aligned}
$$

Then these $t_{k}^{0}$ must be essential vectors. But they depend only on $\partial_{1} z^{\lambda}$ and by hypothesis $E^{\prime} \Sigma$ is complete. Hence $R_{k h}^{1}=0, \lambda_{h}=\lambda_{k}, h>r^{\prime}$. Hence a Jordan basis of $E^{\prime} \Sigma$ is $t_{k}^{s}, 1 \leqq s \leqq m_{k}$ for $1 \leqq k \leqq r^{\prime} ; t_{k}^{s}, 1 \leqq$ $s \leqq m_{k}-1$ for $r^{\prime}<k \leqq r^{\prime \prime} ; w_{k}^{m_{k}}$ for $r^{\prime \prime}<k \leqq r$. Since $\left(A^{\prime}-\lambda_{k} I\right) t_{k}^{s} \equiv$ $t_{k}^{s-1},\left(A^{\prime}-\lambda_{k} I\right) t_{k}^{1} \equiv 0$ and $\left(A^{\prime}-\lambda_{k} I\right) w_{l}^{m_{k}} \equiv 0$, it follows that $\rho\left(E^{\prime} \Sigma\right)=$ $\sum_{k=1}^{r^{\prime}}\left(m_{k}-1\right)+\sum_{k=r^{\prime}+1}^{r^{\prime \prime}}\left(m_{k}-2\right)$. Since some $m_{k}>1$ for $r^{\prime}<k \leqq r^{\prime \prime}$, this number is smaller than $\rho(\Sigma)=\sum_{k=1}^{r \prime \prime}\left(m_{k}-1\right)$. (Recall $m_{k}=1$ for $k>r^{\prime \prime}$.)

Case 2. Every $m_{k}=1$ if $w_{k}^{m} k \notin W(x, z)$. Thus, $E^{\prime} \Sigma$ is obtained by prolonging on the vector fields $w_{k}^{s}, 1 \leqq s \leqq m_{k}, k=1, \cdots, r^{\prime}$, where $m_{k}=1$ for $k>r^{\prime}$. In $\Sigma$ the vectors $w_{k}^{m_{k}}, k>r^{\prime}$ satisfy $\left(A-\lambda_{k} I\right) w_{k}^{m_{k}} \equiv$ 0 . If we prolong $E^{\prime} \Sigma$ on these vector fields $w_{k}^{m}, k>r^{\prime}$, we shall add

$$
z_{k}=w_{k}^{m_{k}}, \partial_{2} z_{k}=\lambda_{k} \partial_{1} z_{k}+T_{k}, k>r^{\prime} .
$$

This in no way alters the degeneracy of $E^{\prime} \Sigma$, whose essential vectors are $\partial_{1} z_{k}^{s}, 1 \leqq s \leqq m_{k}-1$, and whose degeneracy is $\rho(\Sigma)$. Moreover, if we add these equations to each of the systems in the sequence

$$
E^{\prime} \Sigma \subset \Sigma_{1} \subset \cdots \subset \Sigma_{h}
$$


observe that $E^{\prime} \Sigma$ becomes $P \Sigma$. Then since $\Sigma_{1}$ is a $p p$ of $\Sigma$ which contains $E^{\prime} \Sigma$, when we add these equations $\Sigma_{1}$ must have $P \Sigma$ as an admissible restriction. Taking this admissible restriction in each succeeding systems leads to a sequence of $p p^{\prime} \mathrm{s}$

$$
P \Sigma \subset \Sigma_{2}^{\prime} \subset \cdots \subset \Sigma_{h}^{\prime} \text {. }
$$

Now $\Sigma_{j}^{\prime}$ is an admissible restriction of the $p p$ of $\Sigma_{j}$ obtained by adding (8) to $\Sigma_{j}$. The essential vectors of $\Sigma_{j}$ do not depend on $\partial_{1} z^{\lambda}$ since $E^{\prime} \Sigma$ is a complete $p p$ of $\Sigma$, hence they do not depend on $z_{k}$. The vectors $\partial_{1} z_{k}$ are not essential, so adding (8) in no way effects the degeneracy of $\Sigma_{j}$, since degeneracy measures the number of essential vectors. Since admissible restrictions do not effect degeneracy, $\rho\left(\Sigma_{j}^{\prime}\right)=$ $\rho\left(\Sigma_{j}\right)$. In particular $\Sigma_{h}^{\prime}$ is hyperbolic. Finally, use Proposition 25.

THEOREM 4. Let $\Sigma \subset \Sigma_{1} \subset \cdots \subset \Sigma_{h}$ be a sequence of pp's leading to a hyperbolic system $\Sigma_{h}$. Then on an open dense set for some $k$ there is a complete normal pp $E^{\prime} P^{k} \Sigma$ of $P^{k} \Sigma$ having lower degeneracy than $\Sigma$, unless $\Sigma$ is itself hyperbolic.

Proof. By Proposition 25 we can suppose the sequence is normal. By Theorem 2, if $k \geqq m-\alpha_{1}-\rho(\Sigma)+1$ there exists a sequence of $p p$ 's on an open dense set, $P^{k} \Sigma \subset E^{\prime} P^{k} \Sigma \subset \Sigma_{1}^{\prime} \subset \cdots \subset \Sigma_{h}^{\prime}$ where $E^{\prime} P^{k} \Sigma$ is a normal complete $p p$ of $P^{k} \Sigma, \Sigma_{1}^{\prime}$ is a $p p$ of $P^{k} \Sigma$ and $\Sigma_{h}^{\prime}$ is hyperbolic. By Theorem 3 either $\rho\left(E^{\prime} P^{k} \Sigma\right)<\rho(\Sigma)$, in which case the theorem is proved, or else there exists a sequence $P^{k+1} \Sigma \subset \Sigma_{2}^{\prime \prime} \subset \cdots \subset \Sigma_{h}^{\prime \prime}$ of normal $p p^{\prime}$ s where $\Sigma_{h}^{\prime \prime}$ is hyperbolic. Repeating this, there must be some $E^{\prime} P^{j} \Sigma$ of lower degeneracy than $\Sigma$ unless $\Sigma$ is itself hyperbolic.

THEOREM 5. If $\Sigma \subset \Sigma_{1} \subset \cdots \subset \Sigma_{h}$ is a sequence of normal pp's leading to the hyperbolic system $\Sigma_{h}$, then on an open dense set, for all $k \geqq m-\alpha_{1}-\rho(\Sigma)+1$, there exists a complete $p p E^{\prime} P^{k} \Sigma$ such that $\rho\left(E^{\prime} P^{k} \Sigma\right)<\rho(\Sigma)$.

Proof. By Theorem 4 this is true for some $k+j$. By Proposition 24, $\rho\left(E^{\prime} P^{k} \Sigma\right)=\rho\left(P^{j} E^{\prime} P^{k} \Sigma\right)=\rho\left(E^{\prime} P^{k+j} \Sigma\right)<\rho(\Sigma)$.

CoRollary. If $\Sigma$ is absolutely equivalent to a hyperbolic system, then the proceedure in Theorem 2 for successively constructing $E^{\prime} P^{k} \Sigma$ will result in a hyperbolic system. 


\section{REFERENCES}

1. Élie Cartan, Sur l' équivalence absolue de certains systemes d'équations differentielles et sur certaines familles de courbes, Bull. Soc. Math. France 42 (1914) 12-48; Oeuvres Complètes, II, 2, 1133-1168.

2. H. H. Johnson, Absolute equivalence of exterior differential systems, Illinois J. Math. 10 (1966), 407-411.

3. - The non-invariance of hyperbolicity in partial differential equations, Pacific J. Math 22 (1967), 419-430.

Received September 27, 1967.

UNIVERSITY OF WASHINGTON

Seattle, Washington 



\section{PACIFIC JOURNAL OF MATHEMATICS}

\section{EDITORS}

H. ROYDEN

Stanford University

Stanford, California

\section{RichaRd PIERCE}

University of Washington Seattle, Washington 98105
J. DugundJI

Department of Mathematics

University of Southern California

Los Angeles, California 90007

BASIL GORDON

University of California

Los Angeles, California 90024

\section{ASSOCIATE EDITORS}
E. F. BECKENBACH
B. H. NeUMANN
F. WOLF
K. YOSHIDA

\section{SUPPORTING INSTITUTIONS}

UNIVERSITY OF BRITISH COLUMBIA CALIFORNIA INSTITUTE OF TECHNOLOGY

UNIVERSITY OF CALIFORNIA

MONTANA STATE UNIVERSITY

UNIVERSITY OF NEVADA

NEW MEXICO STATE UNIVERSITY

OREGON STATE UNIVERSITY

UNIVERSITY OF OREGON

OSAKA UNIVERSITY

UNIVERSITY OF SOUTHERN CALIFORNIA

\author{
STANFORD UNIVERSITY \\ UNIVERSITY OF TOKYO \\ UNIVERSITY OF UTAH \\ WASHINGTON STATE UNIVERSITY \\ UNIVERSITY OF WASHINGTON \\ AMERICAN MATHEMATICAL SOCIETY \\ CHEVRON RESEARCH CORPORATION \\ TRW SYSTEMS \\ NAVAL WEAPONS CENTER
}

The Supporting Institutions listed above contribute to the cost of publication of this Journal, but they are not owners or publishers and have no responsibility for its content or policies.

Mathematical papers intended for publication in the Pacific Journal of Mathematics should be in typed form or offset-reproduced, double spaced with large margins. Underline Greek letters in red, German in green, and script in blue. The first paragraph or two must be capable of being used separately as a synopsis of the entire paper. It should not contain references to the bibliography. Manuscripts, in duplicate if possible, may be sent to any one of the four editors. Please classify according to the scheme of Math. Rev. 36, 1539-1546. All other communications to the editors should be addressed to the managing editor, Richard Arens, University of California, Los Angeles, California, 90024.

50 reprints are provided free for each article; additional copies may be obtained at cost in multiples of 50 .

The Pacific Journal of Mathematics is published monthly. Effective with Volume 16 the price per volume (3 numbers) is $\$ 8.00$; single issues, $\$ 3.00$. Special price for current issues to individual faculty members of supporting institutions and to individual members of the American Mathematical Society: $\$ 4.00$ per volume; single issues $\$ 1.50$. Back numbers are available.

Subscriptions, orders for back numbers, and changes of address should be sent to Pacific Journal of Mathematics, 103 Highland Boulevard, Berkeley, California, 94708.

PUBLISHED BY PACIFIC JOURNAL OF MATHEMATICS, A NON-PROFIT CORPORATION

Printed at Kokusai Bunken Insatsusha (International Academic Printing Co., Ltd.), 7-17. Fujimi 2-chome, Chiyoda-ku, Tokyo, Japan. 


\section{Pacific Journal of Mathematics}

\section{Vol. 30, No. $3 \quad$ November, 1969}

Willard Ellis Baxter, Topological rings with property $(Y) \ldots \ldots \ldots \ldots . \ldots 5$

Sterling K. Berberian, Note on some spectral inequalities of $C . R$.

Putnam ..................................... 573

David Theodore Brown, Galois theory for Banach algebras . ........... 577

Dennis K. Burke and R. A. Stoltenberg, A note on p-spaces and Moore spaces ........................................ 601

Rafael Van Severen Chacon and Stephen Allan McGrath, Estimates of positive contractions....................................... 609

Rene Felix Dennemeyer, Conjugate surfaces for multiple integral problems in the calculus of variations ........................... 621

Edwin O. Elliott, Measures on countable product spaces.............. 639

John Moss Grover, Covering groups of groups of Lie type .............. 645

Charles Lemuel Hagopian, Concerning semi-local-connectedness and cutting in nonlocally connected continua .................. 657

Velmer B. Headley, A monotonicity principle for eigenvalues ........... 663

John Joseph Hutchinson, Intrinsic extensions of rings . . . . . . . . . . . . . 669

Harold H. Johnson, Determination of hyperbolicity by partial

prolongations .................................. 679

Tilla Weinstein, Holomorphic quadratic differentials on surfaces in $E^{3} \ldots 697$

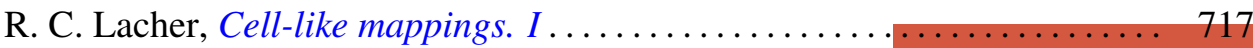

Roger McCann, A classification of centers

Curtis L. Outlaw, Mean value iteration of nonexpansive mappings in a

Banach space...

Allan C. Peterson, Distribution of zeros of solutions of a fourth order

differential equation.

Bhalchandra B. Phadke, Polyhedron inequality and strict convexity .. 765 Jack Wyndall Rogers Jr., On universal tree-like continua .

Edgar Andrews Rutter, Two characterizations of quasi-Frobenius rings

G. Sankaranarayanan and C. Suyambulingom, Some renewal theorems concerning a sequence of correlated random variables...

Joel E. Schneider, A note on the theory of primes........ . .

Richard Peter Stanley, Zero square rings .................

Edward D. Tymchatyn, The 2-cell as a partially ordered space

Craig A. Wood, On general Z.P.I.-rings................ 